\title{
A 3D Statistical Shape Model for the Left Ventricle of the Heart
}

\author{
Hui Luo ${ }^{1}$ and Thomas O’Donnell ${ }^{2}$ \\ ${ }^{1}$ Dept. of CS and Engineering, SUNY Buffalo, Amherst, NY 14260 \\ huiluo@cse.buffalo.edu \\ ${ }^{2}$ Siemens Corporate Research, Princeton, NJ 08540 \\ odonnell@scr.siemens.com
}

\begin{abstract}
We propose a method for creating a 3-D statistical shape model of the left ventricle from sets of sparse 2-D contour inputs. Since the input contours may not delineate consistent portions of the underlying organ from subject to subject, we apply a model-based approach to make associations (landmarks) between training examples. Included is a measure of confidence in these created landmarks. Statistics on the model shape are garnered via principal component analysis. The results of applying the technique to a 14 subject database are presented.
\end{abstract}

In this paper we describe a method for developing a statistical shape model of the left ventricle (LV) of the heart. We assume the existence of a set of segmented LV instances culled from both patient and volunteer Magnetic Resonance (MR) datasets. The LVs were segmented using the Argus ${ }^{\mathrm{TM}}$ tool which draws 2-D contours on individual image slices. Each MR dataset typically has six to seven short axis slices as well as at least one long-axis slice. The datasets provide sparse but adequate coverage of the LV. Note that the positions of the image slices with respect to the LV vary from dataset to dataset. Therefore contours may delineate different sections of the LV and cannot be used directly as landmarks.

Given these inputs, there are two major issues in developing a statistical model of the LV. First, employing a user-defined landmark-based approach can be challenging as well as time consuming because there are very few distinct features in the underlying LV on which to specify such points. Second, since the landmarks may not be directly delineated by the contours, the concept in confidence in the landmarks must be incorporated.

To address the first concern, we manufacture a dense set of landmarks in a semiautomatic way. As mentioned, the LV has very few distinct, easily labeled points; however, there are several overall shape similarities expressed as regions of curvature. We use a model-based approach to interpolate between sparse contours and align the models using the overall shape [O'Donnel100]. Following this we create a dense set of new associations (landmarks) between the model surfaces based on a distance metric. Addressing the second concern, the landmarks are averaged using a weighted sum based on a landmark's proximity to its contours. Finally we create a statistical shape model by principle component analysis using these landmarks. 
Work closest to ours focuses on the problem of generating dense set of landmarks semi-automatically [Lorenz00, Fleute98, Brett00, Park95]. We view our contributions as: 1) a model-based alignment for sparse inputs. 2) the weighting of landmarks which appropriately describes the reliability of each landmark's situation.

Briefly, our approach is to create a template model, align the datasets making use of this template, fit models to each of the aligned datasets, make associations (landmarks) between the model surfaces (again using the template model), and average those landmarks to create a Procrustes mean shape.

The proposed method has been used to generate 3D statistical LV model from pool of 14 subjects. Table 1 illustrates the relative contribution of the most influential components. From this we can see that nearly $90 \%$ of the shape variation can be captured by the first nine eigenvectors.

Table 1. Relative contributions of eigenvectors.

\begin{tabular}{|c|c|c|}
\hline Index & $\left(\lambda_{\mathrm{i}} / \lambda_{\text {total }}\right)$ & Cumulative total \\
\hline 1 & $41.3769 \%$ & $41.3769 \%$ \\
\hline 2 & $10.4376 \%$ & $51.8144 \%$ \\
\hline 3 & $9.0261 \%$ & $60.8406 \%$ \\
\hline 4 & $7.5134 \%$ & $68.3540 \%$ \\
\hline 5 & $6.2111 \%$ & $74.5651 \%$ \\
\hline 6 & $4.0443 \%$ & $78.6094 \%$ \\
\hline 7 & $3.9312 \%$ & $82.5406 \%$ \\
\hline 8 & $3.4228 \%$ & $85.9634 \%$ \\
\hline 9 & $3.0939 \%$ & $89.0573 \%$ \\
\hline
\end{tabular}
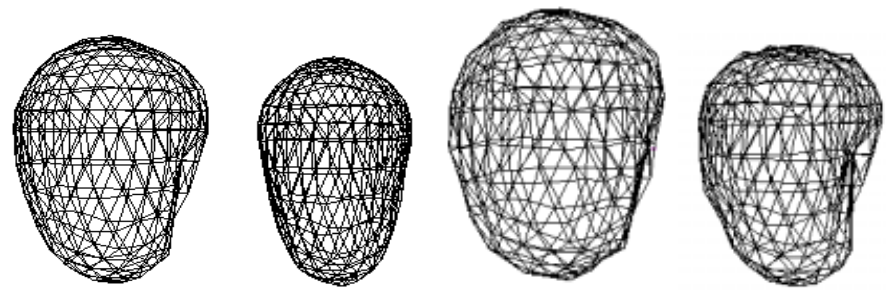

Fig. 1. Leftmost two: The epicardial and endocardial surfaces of the average model. Rightmost two: The result of varying the first mode of variation by three standard deviations.

\section{References}

[Fleute98] Fleute M, et. al.,Building a complete surface model from sparse data using statistical shape models: application to computer assisted knee surgery. MICCAI'98 1998, pp.879-87.

[Lorenz00] Lorenz C, Krahnstover N. Generation of point-based 3D statistical shape models for anatomical objects. CVIU, vol.77, no.2, Feb. 2000, pp.175-91.

[Brett00] Brett AD, Taylor CJ. A method of automated landmark generation for automated 3D PDM construction. Image \& Vision Computing, vol.18, no.9, June 2000, pp.739-48.

[O’Donnell00] O'Donnell T, et. al., Multi Modality Model-Based Registration. CVPROO.

[Park95] Park J, Metaxas D, et. al., Deformable Models with parameter Functions. CVPR95. 